\title{
Optical and Reflective Characteristics Measurement and Focal Length Calculation of a Hollow Tetrahedral Corner and a Solid Highly Retroreflective Cubes
}

\author{
Mohammad Saeed Marouf (D) \\ Laboratory of Laser Technology, Higher Institute for Laser Researches and Applications, Damascus University, Syria \\ Correspondence should be addressed to Mohammad Saeed Marouf; saeedmaroof123@gmail.com
}

Received 14 March 2019; Revised 26 May 2019; Accepted 16 June 2019; Published 15 July 2019

Academic Editor: Wonho Jhe

Copyright (C) 2019 Mohammad Saeed Marouf. This is an open access article distributed under the Creative Commons Attribution License, which permits unrestricted use, distribution, and reproduction in any medium, provided the original work is properly cited.

Corner cubes are one of the most important optical tools used in new optical devices and optical LIDAR. This paper compares two different designs of the hollow and solid tetrahedral corner cubes and determines the relation between the Retroreflection index and the surface quality $\mathrm{N}$ and the surface flatness $\Delta \mathrm{N}$ and its effect on the focal length of the hollow and solid corner cube.

\section{Introduction}

The technology of hollow and solid tetrahedral optical corner cubes has received a great attention in recent years due to its use in the design of optical technologies, optical LIDAR, and optical measurements. The efforts have increased recently to develop light and high performance corner cubes free from optical and geometrical defects, to be used in the advanced measurements, precision measurement devices, remote sensing, astronomical measurements, traffic figures, airports, and other engineering applications.

Changes in Retroreflection as a function to the optical corner cubes' surface quality is discussed. And the focal length of the optical corner cube and its relationship with Retroreflection index during manufacturing phases are investigated.

Purpose of the Research. This work aims at specifying the conditions that allow evaluating the performance of corner cube retroreflectors for long distance without laser beam dispersion and establishing the effect of roughness and flatness $(\mathrm{N}, \Delta \mathrm{N})$ on the coefficient of optical Retroreflection.

\section{Retroreflection Index Change as a Function of $N$ and $\Delta N$}

$\mathrm{N}$ : the number of Newton rings (surface quality) of back surfaces of tetrahedral corner cube.

$\Delta \mathrm{N}$ : flatness factor of back surfaces.

P-V: peak of valley $=2 . \Delta \mathrm{N}$.

The change in Retroreflection index has been measured as a function of $\mathrm{N}$ and $\Delta \mathrm{N}$ during the surface finishing process, where the front surface (incidence face) has been completely finished with high precision $[1,2]$

$(\mathrm{N}=0.196, \Delta \mathrm{N}=0.2)$, as it is shown in Figure 1.

Note that the high precision of surface orthogonality and quality and flatness factors of surfaces have been taken into account in the design chart and that interference photos and measurements have been taken for each step as it is shown in Figures 2(a), 2(b), 2(c), and 2(d).

(a) Figure 2(a) shows the quality and flatness factors of lateral surfaces of tetrahedral optical corner cube.

(b) Figure 2(b) shows the quality and flatness factors of front surfaces of tetrahedral optical corner cube. 


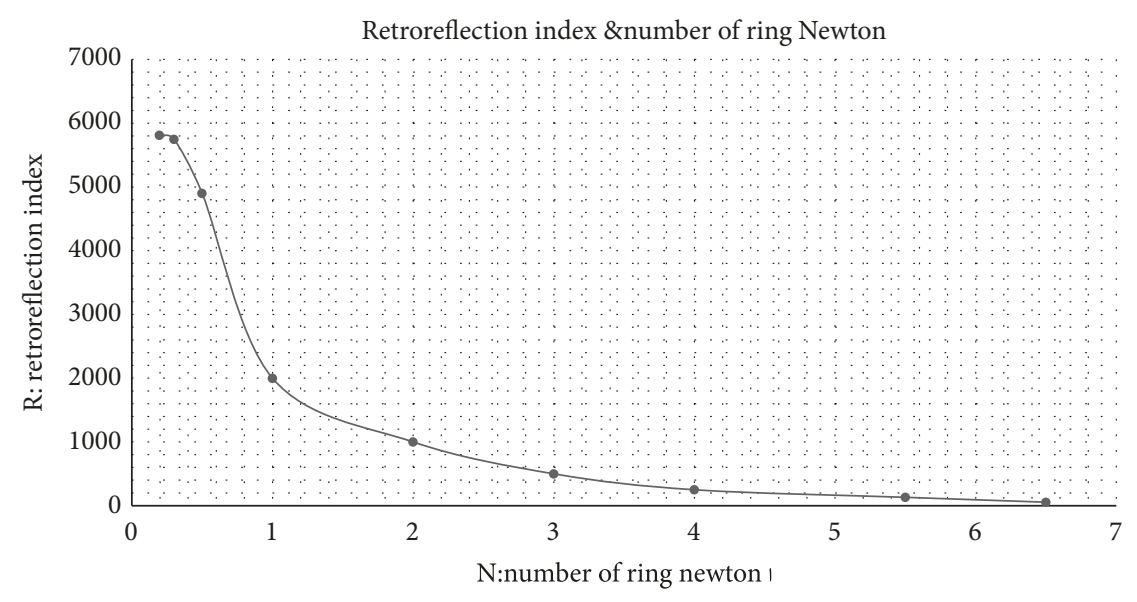

FIgURE 1: The change in Retroreflection index as a function of $\mathrm{N}$.

(c) Figure 2(c) shows the interference between the six sectors of tetrahedral corner cube.

(d) Figure 2(d) shows the tetrahedral defects during the manufacturing process. As we can see from the figure, there are two sectors that are more opaque than the others, and this is one of the common defects that appear during the manufacturing process of corner cubes and prisms in general. This is because of noncomplete orthogonality between two of the back surfaces of corner cube, and when this corner cube is to be used in the laser chamber we obtain a dual or trial laser pulse, and this in turn increases the pulse width. Also, when the corner cube is to be used in optical LIDAR, we will obtain more than one spot back from the corner cube, and this in turn decreases the intensity of the main light spot $[3,4]$.

(e) The interference rings fringes were measured using the Fizeau device (manufactured by MOLLER) as it is shown in Figure 2(e).

\section{Calculation of Focal Length of Solid Tetrahedral Corner Cube}

When the reflection sides and incident face are flat and manufactured using one piece of glass and when using the design and technical specifications that lead usually to highly precise spherical surfaces, then the corner cube will have an aberrant optical system such as the other known optical environments with concavity radius $r$. In this case the corner cube will have a focal length $\mathrm{f}$ and power $\phi$, where $\phi=1 / \mathrm{f}$ is approximately calculated as follows $[4,5]$ :

$$
\phi=\frac{1}{\mathrm{r}_{\mathrm{i}}}+5\left(\frac{1}{r_{a}}+\frac{1}{r_{b}}+\frac{1}{r_{c}}\right)
$$

where

$r_{i}$ is the concavity radius of incident face of corner cube;

$r_{a}, r_{b}, r_{c}$ are the concavity radiuses of other side faces of corner cube.
The value of the focal length may reach many hundreds or thousands of meters when the optical faces are manufactured with high precision. For simplification in designing the geometrical shape, the diameter of the one reflected beam at the source level (without taking the diffraction phenomena into account), for a focal length $\mathrm{f}$ of the corner cube, is calculated using the following formula [4-7]:

$$
D_{1}=D_{0}+d\left|\frac{L}{f}-2\right|
$$

For $\mathrm{L} \gg \mathrm{f}$, the equation above becomes as follows:

$$
D_{1}=\mathrm{D}_{0}+\frac{\mathrm{dL}}{\mathrm{f}}
$$

Example 1. $\mathrm{L}=2.5 \mathrm{Km}, \mathrm{d}=0.25 \mathrm{~m}, \mathrm{D}_{0}=0.5 \mathrm{~cm}, \mathrm{f}=500 \mathrm{~m}$, and then $\mathrm{D}_{1}=1.255 \mathrm{~m}$.

For the case of large $\mathrm{L}$, the reflected beam diameter is determined from the following relation that combines the focal length and the diffraction phenomena:

$$
D_{2}=L\left(\frac{d}{f}+1.2 \frac{\lambda}{d}\right)
$$

The illumination and Retroreflection index resulted from the reflection of the light beam have a maximum value at the case of normal incidence of rays on the incidence face of corner cube.

First we will calculate the concavity radius of front face and lateral sides of the corner cube. Figure 3 shows how to calculate this radius. by

For a number of Newton rings $\mathrm{N}=6.5$, the depth $\delta$ is given

$\delta=\mathrm{N} . \lambda / 2=6.5 . \lambda / 2=1,625 \cdot 10^{-6} \mathrm{~m}$, where the chosen wavelength is the middle of the visible region $=0,5 \mu \mathrm{m} \lambda$.

Supposing D is the diameter of the active orifice of the light from the flat surface, then the needed concavity radius $r$ is given by

$$
\mathrm{r}^{2}=\left(\frac{\mathrm{D}}{2}\right)^{2}+(\mathrm{r}-\delta)^{2}
$$




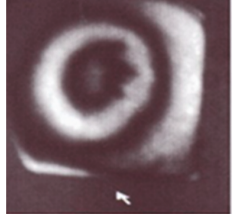

$\mathrm{N}=-2$

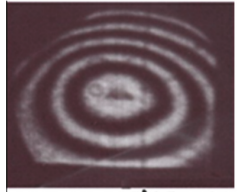

$\mathrm{N}=-4 \quad \Delta \mathrm{N}=1.94$

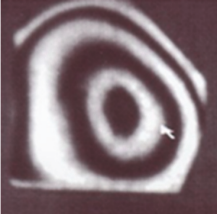

$\mathrm{N}=-3$

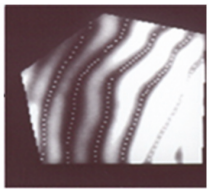

$\mathrm{N}=-0.28$

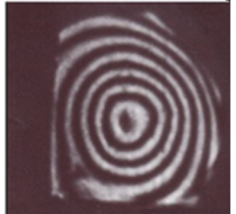

$\mathrm{N}=-5.5$

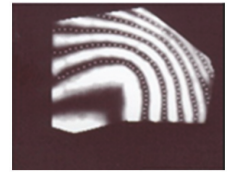

$\mathrm{N}=-2.92$

$\Delta \mathrm{N}=1.14$

The interstitial picture of the entire surface

(a) The quality and flatness factors of lateral surfaces of tetrahedral optical corner cube

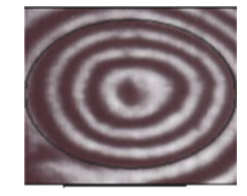

$\mathrm{N}=-5.5$

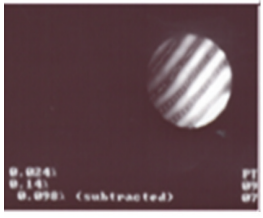

$\mathrm{N}=-0.196$

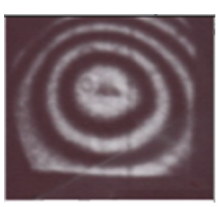

$\mathrm{N}=-4$

Front surface

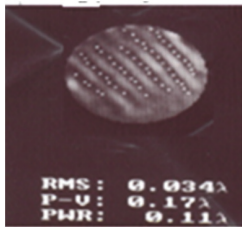

$\mathrm{N}=-0.22$

Front surface

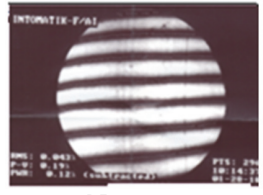

$\mathrm{N}=-0.2$

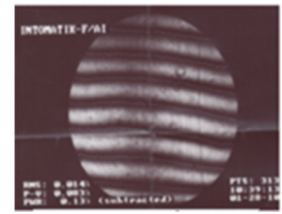

$\mathrm{N}=-2$

(b) The quality and flatness factors of front surfaces of tetrahedral optical corner cube
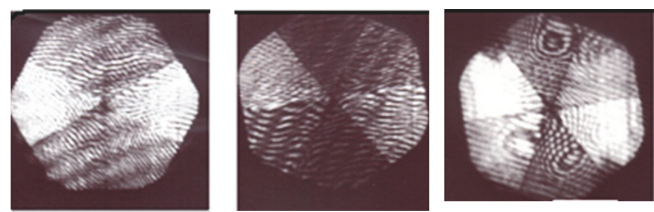

$\mathrm{N}=-3 \quad \Delta \mathrm{N}=2$

$\mathrm{N}=-1.5 \quad \Delta \mathrm{N}=1.12$

$\mathrm{N}=-5 \quad \Delta \mathrm{N}=5$

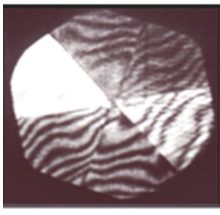

$\mathrm{N}=-1 \quad \Delta \mathrm{N}=0.5$

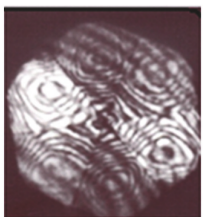

$\mathrm{N}=-4 \quad \Delta \mathrm{N}=3$

Front surface

Number of Newton ring between photoreceptor sectors

(c) The interference between the six sectors of tetrahedral corner cube

Figure 2: Continued. 

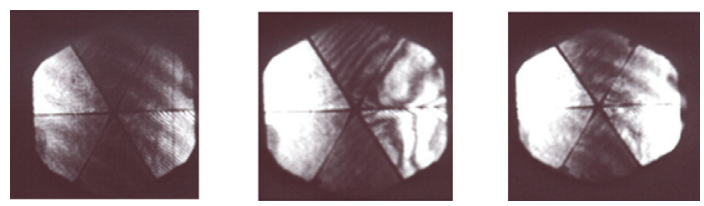

(d) Tetrahedral defects during the manufacturing process of corner cubes

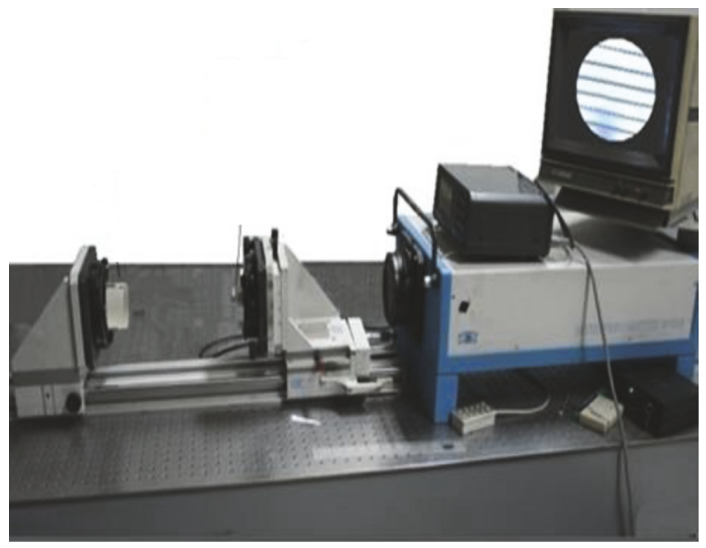

(e) Fizeau Interferometer device

Figure 2

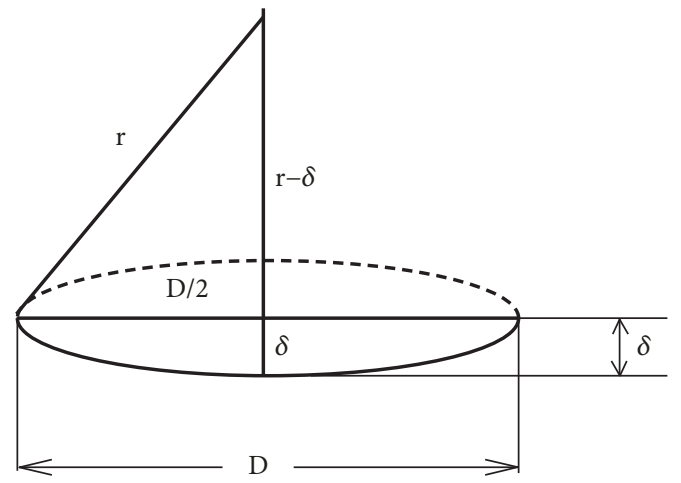

FIgURE 3: The concavity radius $r$ and its relation to number of Newton rings $\mathrm{N}$ of optical surface [7,9]. D: the diameter of the effective aperture of light from the flat surface and $\delta$ : the distorted part of the level surface.

By simplification, the equation above becomes

$$
r=\frac{D^{2}}{8 \delta}+\frac{\delta}{2}
$$

When the used space of the flat surface is a circle with a diameter $\mathrm{D}=60 \mathrm{~mm}=60.10^{-3} \mathrm{~m}$, then $\mathrm{r}=\mathrm{r}_{\mathrm{i}}$ for the incidence face of the solid corner cube.

$$
r_{i}=360 \mathrm{~m}
$$

Also we calculate the concavity radiuses $r_{a}, r_{b}$, and $r_{c}$ for the three lateral surfaces with depths $\delta_{1}=\delta_{2}=\delta_{3}=\mathrm{N} .(\lambda / 2)$, respectively, so if the light spot on the lateral surfaces is $\mathrm{D}_{\mathrm{a}}=$ $\mathrm{D}_{\mathrm{b}}=\mathrm{D}_{\mathrm{c}}=50 \mathrm{~mm}$, then the concavity radiuses will be $r_{a}=r_{b}=r_{c}=250 m$. Substituting in relation (1) taking into account the light incidence direction and the concavity direction at each surface we obtain

$$
\phi=\frac{1}{f} \Longrightarrow f=-81.81 m
$$

where $\phi$ is the power of the lens in $\mathrm{m}^{-1}$ or diopter.

This means that, for a large number of rings $\mathrm{N}=6.5$, the corner cube is a bad one and the beam will diverge after a short distance equal to $\mathrm{f}=-81.81 \mathrm{~m}$. However, for $\mathrm{N}$ $=0.196 \mathrm{f}$ becomes equal to $\mathrm{f}=-1269 \mathrm{~m}$, so the corner cube in this case will be equivalent to a negative lens with large focal length, which is very important in the applications that require large distances measurements such as leader. For the case of astronomic measurements, there will be a need to adhere additional lens to the corner cube in such a manner that the focal length increases to assure the nondivergence of the light beam coming back from the corner cube. In this case, the focal lengths of the corner cube and the additional lens are summed together according to the known lens laws. Table 1 shows the number of Newton rings and focal length for each of the cases discussed above.

We conclude that the lesser the number of Newton rings $\mathrm{N}$, the larger the concavity radius $\mathrm{r}$, and the smaller the power $\phi$. This means that the focal length, which represents the corner cube, becomes larger. As a result, the light beam coming back from the corner cube will not focus rapidly, which is very useful in applications that need large distances (far field). One can draw a curve of the focal length $f[\mathrm{~m}]$ versus the number of Newton rings $\mathrm{N}$, as it is shown in Figure 4. From this figure, we can see how the focal length increases with the decrease in number of rings. Also one can 
TABLE 1: The number of Newton rings and focal length for each case individually.

\begin{tabular}{lcccccccc}
\hline $\mathrm{N}$ & $\mathrm{r}_{\mathrm{i}}[\mathrm{m}]$ & $\mathrm{r}_{\mathrm{a}}=\mathrm{r}_{\mathrm{b}}=\mathrm{r}_{\mathrm{c}}[\mathrm{m}]$ & $\Delta \mathrm{N}$ & $\mathrm{D}[\mathrm{mm}]$ & $\delta[\mu]$ & $\mathrm{R}\left[\mathrm{m}^{2} / \mathrm{str}\right]$ & $\Phi\left[\mathrm{m}^{-1}\right]$ & $\mathrm{f}[\mathrm{m}]$ \\
\hline 6.5 & 360 & 250 & 2.2 & 50 & 1.25 & 54.3 & -0.01222 & -81.81 \\
\hline 5.5 & 450 & 312 & 2.0 & 50 & 1 & 130.9 & -0.0078 & -113 \\
\hline 4 & 600 & 500 & 1.8 & 50 & 0.75 & 249.4 & 0.00834 & -119.7 \\
\hline 3 & 900 & 625 & 1.5 & 50 & 0.5 & 499.4 & -0.0069 & -144 \\
\hline 2 & 1800 & 1250 & 1.14 & 50 & 0.25 & 999.4 & -0.0035 & -285 \\
\hline 1 & 2500 & 2000 & 1.0 & 50 & 0.5 & 1999.5 & -0.0021 & -476 \\
\hline 0.5 & 3600 & 2500 & 0.5 & 50 & 0.125 & 4899.4 & -0.00172 & -580.6 \\
\hline 0.3 & 6000 & 4500 & 0.2 & 50 & 0.075 & 5749.4 & -0.00095 & -1052 \\
\hline 0.196 & 7250 & 5400 & 0.2 & 50 & 0.05 & 5812 & -0.00078 & -1269 \\
\hline
\end{tabular}

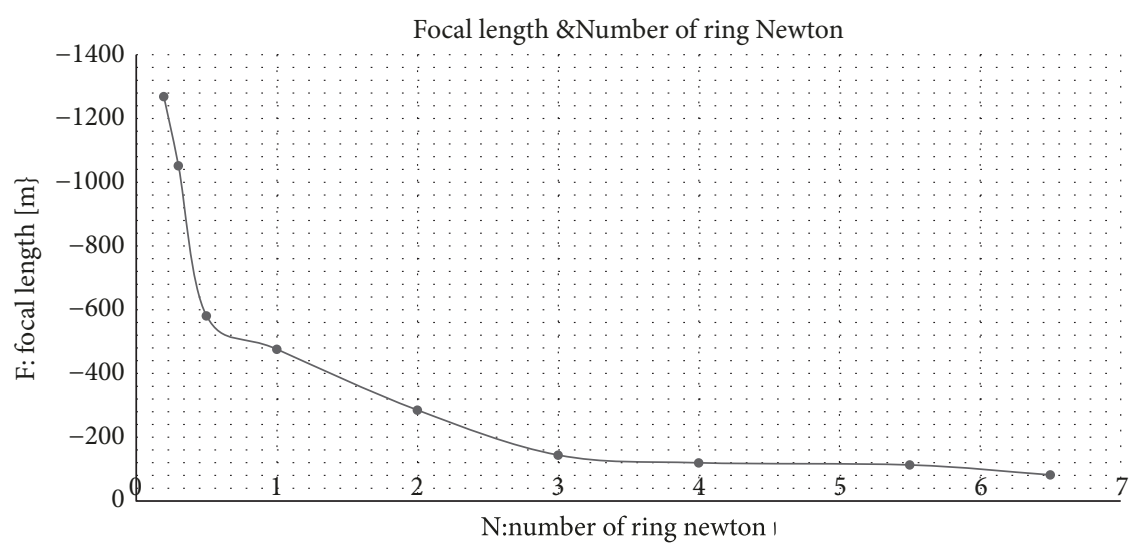

Figure 4: The focal length $\mathbf{f}[\mathrm{m}]$ versus the number of Newton rings $\mathrm{N}$.

draw a curve of the Retroreflection index $\mathrm{R}\left[\mathrm{M}^{2} / \mathrm{Str}\right]$ versus the focal length of the corner cube $\mathrm{f}[\mathrm{m}]$, as it is shown in Figure 5.

\section{Calculation of Focal Length of Hollow Tetrahedral Corner Cube}

When the reflection sides are flat and manufactured using one piece of glass and when using the design and technical specifications that lead usually to high precise spherical surfaces, then the corner cube will have an aberrant optical system such as the other known optical environments with concavity radius $\mathbf{r}$. In this case the corner cube will have a focal length $\mathrm{f}$ and power $\phi$, where $\phi=\mathbf{1} / \mathrm{f}$ is approximately calculated as follows [4-8]:

$$
\phi=5\left(\frac{1}{r_{a}}+\frac{1}{r_{b}}+\frac{1}{r_{c}}\right)
$$

where

$r_{a}, r_{b}$, and $r_{c}$ are the concavity radiuses of other side faces of corner cube.

The value of the focal length may reach many hundreds or thousands of meters when the optical faces are manufactured with high precision. For simplification in designing the geometrical shape, the diameter of the one reflected beam at the source level (without taking the diffraction phenomena into account), for a focal length $\mathrm{f}$ of the corner cube, is calculated using the same formulas used for the solid tetrahedral corner cube.

Also we calculate the concavity radiuses $r_{a}, r_{b}$, and $r_{c}$ for the three lateral surfaces with depths $\delta_{1}=\delta_{2}=\delta_{3}=\mathrm{N} \cdot(\lambda / 2)$, respectively, so if the light spot on the lateral surfaces is $\mathrm{D}_{\mathrm{a}}=$ $\mathrm{D}_{\mathrm{b}}=\mathrm{D}_{\mathrm{c}}=50 \mathrm{~mm}$, then the concavity radiuses will be

$r_{a}=r_{b}=r_{c}=250 m$. Substituting in relation (9) taking into account the light incidence direction and the concavity direction at each surface, we obtain

$$
\phi=\frac{1}{f} \Longrightarrow f=-83.75 m
$$

where $\phi$ is the power of the lens in $\mathrm{m}^{-1}$ or diopter.

This means that, for a large number of Newton rings $\mathrm{N}=$ 6.5 , the corner cube is a bad one and the beam will diverge after a short distance equal to $\mathrm{f}=-83.75 \mathrm{~m}$. However, for $\mathrm{N}$ $=0.196 \mathrm{f}$ becomes equal to $\mathrm{f}=-1960.9 \mathrm{~m}$, so the corner cube in this case will be equivalent to a negative lens with large focal length, which is very important in the applications that require large distances measurements such as LIDAR. For the case of astronomic measurements, there will be a need to adhere additional lens to the corner cube in such a manner that the focal length increases to assure the nondivergence of the light beam coming back from the corner cube. In this case, the focal lengths of the corner cube and the additional lens are summed together according to the known lens laws. Table 2 
TABLE 2: The number of Newton ring and focal length for each case individually.

\begin{tabular}{|c|c|c|c|c|c|c|c|}
\hline $\mathrm{N}$ & $r_{a}=r_{b}=r_{c}[m]$ & $\Delta \mathrm{N}$ & $\mathrm{D}[\mathrm{mm}]$ & $\delta[\mu]$ & $\mathrm{R}\left[\mathrm{m}^{2} / \mathrm{str}\right]$ & $\Phi\left[\mathrm{m}^{-1}\right]$ & $\mathrm{f}[\mathrm{m}]$ \\
\hline 6.5 & 250 & 2.2 & 50 & 1.25 & 60.3 & -0.01194 & -83.75 \\
\hline 5.5 & 312 & 2.0 & 50 & 1 & 135.19 & -0.00557 & -179.2 \\
\hline 4 & 500 & 1.8 & 50 & 0.75 & 300.14 & -0.00511 & -195.6 \\
\hline 3 & 625 & 1.5 & 50 & 0.5 & 600.55 & -0.00410 & -243.9 \\
\hline 2 & 1250 & 1.14 & 50 & 0.25 & 2500.21 & -0.00300 & -300.0 \\
\hline 1 & 2000 & 1.0 & 50 & 0.5 & 3200 & -0.00203 & -492.6 \\
\hline 0.5 & 2500 & 0.5 & 50 & 0.125 & 5120 & -0.00112 & -892.8 \\
\hline 0.3 & 4500 & 0.2 & 50 & 0.075 & 6212 & -0.00081 & -1234 \\
\hline 0.196 & 5400 & 0.2 & 50 & 0.05 & 6852 & -0.00051 & -1960 \\
\hline
\end{tabular}

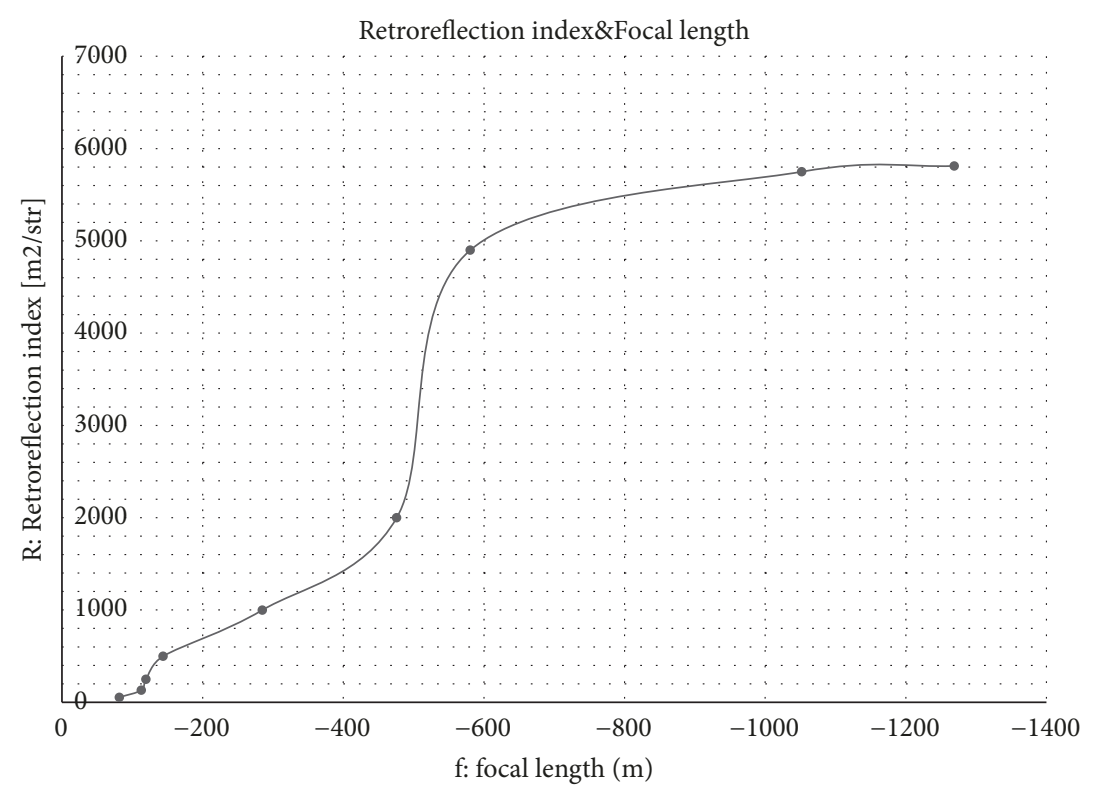

Figure 5: The Retroreflection index $\mathbf{R}\left[\mathrm{M}^{2} / \mathrm{Str}\right]$ versus the focal length $\mathbf{f}$.

shows the number of rings and focal length for each of the cases discussed above.

We conclude that if the number of Newton rings $\mathrm{N}$ is small the concavity radius $\mathrm{r}$ is large, and the power $\phi$ is small. This means that the focal length, which represents the corner cube, becomes larger. As a result the light beam coming back from the corner cube will not focus rapidly, which is very useful in applications that need large distances (far field). One can draw a curve of the focal length $\mathrm{f}[\mathrm{m}]$ versus the number of Newton rings $\mathrm{N}$, as it is shown in Figure 6. From this figure, we can see how the focal length increases with the decrease in number of rings. Also one can draw a curve of the Retroreflection index $\mathrm{R}\left[\mathrm{M}^{2} / \mathrm{Str}\right]$ versus the focal length of the corner cube $\mathrm{f}[\mathrm{m}]$, as it is shown in Figure 7.

\section{Conclusion}

The study of the corner cubes has helped as a mean of choosing the tetrahedral corner cube as an optical tool that deserves investigation. In studying the different factors affecting the Retroreflection index of this tool, we have concluded the following:

(i) There is a difference in the Retroreflection index between the corner cube coated on the back surface and the noncoated one.

(ii) The glass type does not affect the Retroreflection index in the case of a back coated corner cube.

(iii) The two factors $\mathrm{N}$ and $\Delta \mathrm{N}$ affect the Retroreflection index. Form the studied Figures 2(a), 2(b), 2(c), and $2(\mathrm{~d})$ we conclude that the Retroreflection index increases with the increases in each of the surface quality $\mathrm{N}$ and the surface flatness $\Delta \mathrm{N}$. This is because the roughness causes light diffraction, which in turn reduces the amount of the retroflexed light. Also the sphericity of the surface make it plays the role of a negative lens for the large distances. For a large N, the sphericity of the surface increases, so it will play the role of a lens, and the total three reflections seem as the pass of light through many lenses, which in 


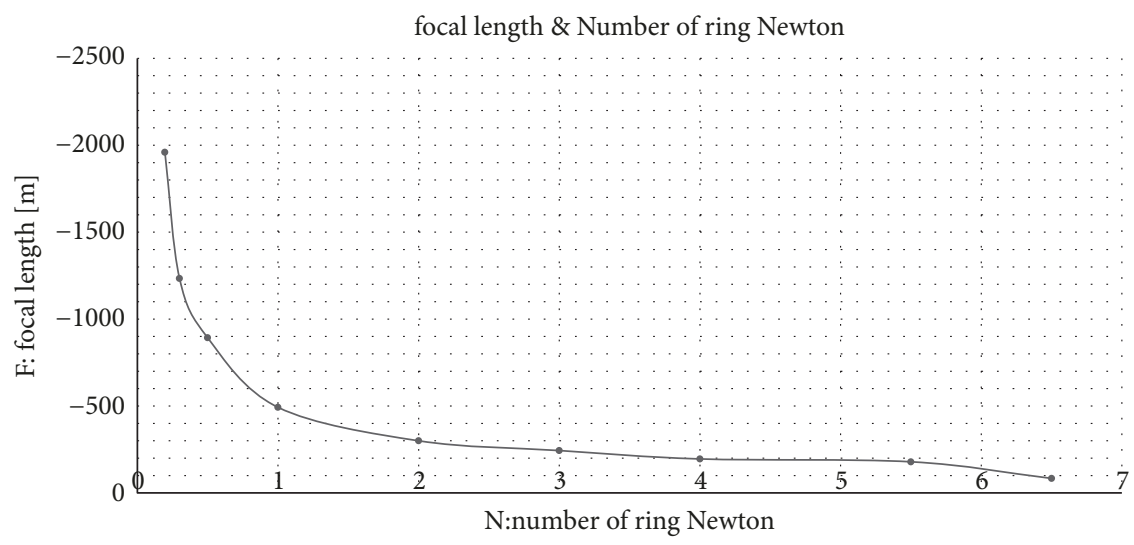

Figure 6: The focal length $\mathbf{f}[\mathrm{m}]$ versus the number of Newton rings $\mathbf{N}$.

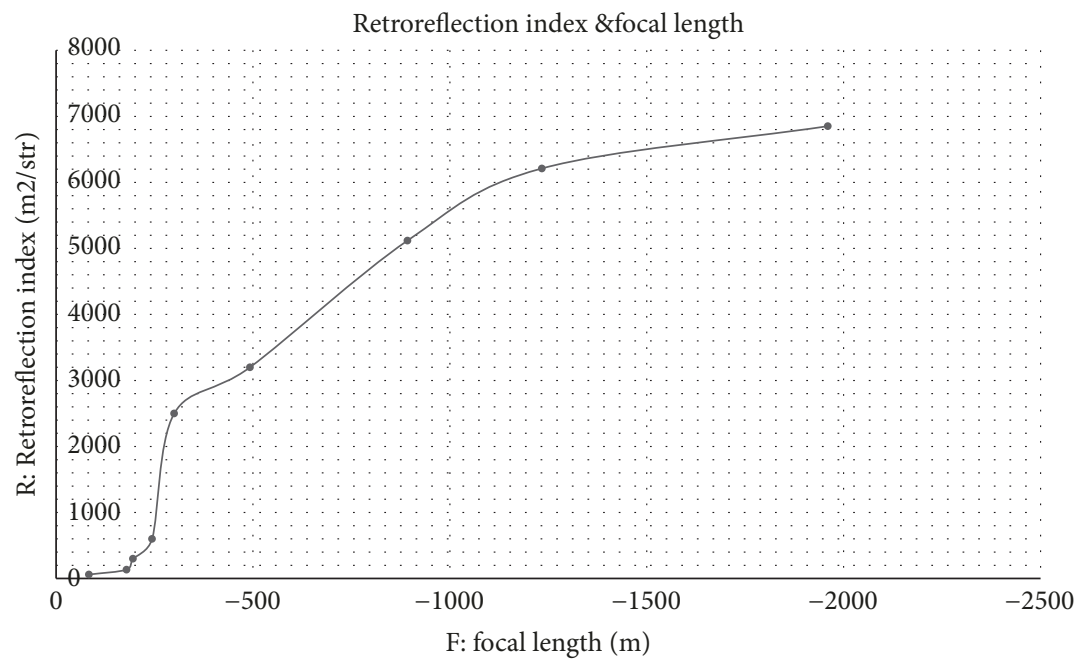

FIGURE 7: The Retroreflection index $\mathbf{R}\left[\mathrm{M}^{2} / \mathrm{Str}\right]$ versus the focal length $\mathbf{f}[\mathrm{m}]$.

turn will result in focusing the laser beam coming back from the corner cube in the case of large flatness factor. The solution of this problem is to make the surface flatness factor as small as possible during the surface finishing processes of corner cubes, using high precise standard reference surfaces during the inspection and check processes of the quality and flatness of the surfaces. For the case of hollow corner cube, each of the optical surfaces is considered as a spherical refractor, which results also in reaching the Retroreflection light to larger distances than the case of solid corner cube. However, the difficulty in this case is in making the surfaces orthogonal to each other.

\section{Data Availability}

The detailed experimental data used to support the findings of this study are available from the corresponding author upon request

\section{Conflicts of Interest}

The author declares that they have no conflicts of interest.

\section{Acknowledgments}

The author would like to thank Dr. M. Sayem El-Daher of the higher Institute for laser research at Damascus University and Eng. Hisham Alhorani for the assistance they have provided to this work.

\section{References}

[1] K. Rachel, Broadband on Rails: A Compact Lens Could Make High-Speed Internet Access Commonplace on Trains, MIT Technology-Eview, 2009.

[2] G. H. Seward, "Measurement and characterization of angular reflectance for cube-corners and microspheres," Optical Engineering, vol. 38, no. 1, pp. 164-169, 1999.

[3] J. Liu and R. M. A. Azzam, "Polarization properties of corner cube retro reflector: theory and experimen," Applied Optics, vol. 36, no. 7, 1997. 
[4] "Visual specifications of pyramidal visual referents," Research Paper 535.318,531.719.24, 1988 (Russian), н.э.ритынь.

[5] "The formation and technology of reflective light reflectors" (Russian), г.в.денисюк.в.и.корнеев535.513, 1991.

[6] S. Marouf, Z. Moussa, and F. Saiouf, Design and Realization of a Solid Corner Cube and a Spherical Retroreflector and Measurement of Their Optical and Retro-Reflective Properties, HILRA, Damascus University, Syria, 2011.

[7] S. Marouf, Z. Moussa, and F. Saiouf, The Retroreflection Index of Optical and Opto-Electronical Systems, HILRA, Damascus university, Syria, 2011.

[8] N. Hodgson and H. Weber, Laser Resonators and Beam Propagation, Chapter 17, Springer, 2nd edition, 2005.

[9] K. Vedam and M. D. Stoudt, "Retroreflection from spherical glass beads in highway pavement markings. 2: diffuse reflection (a first approximation calculation)," Applied Optics, vol. 17, no. 12, pp. 1859-1869, 1978. 

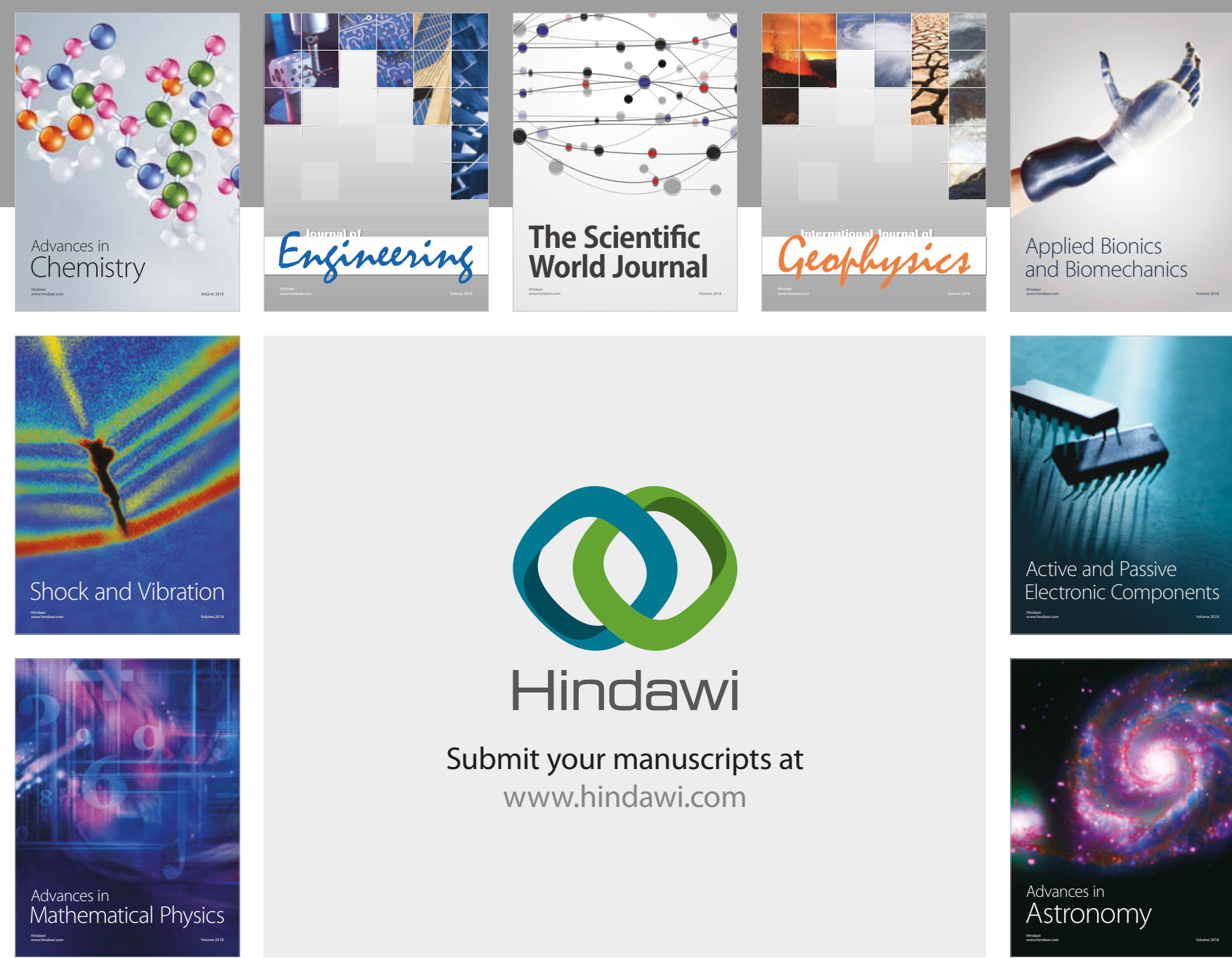

Submit your manuscripts at

www.hindawi.com

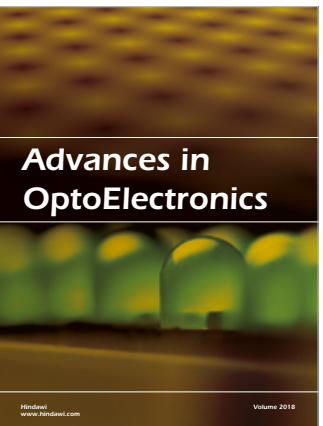

\section{Rotcting Machinery}
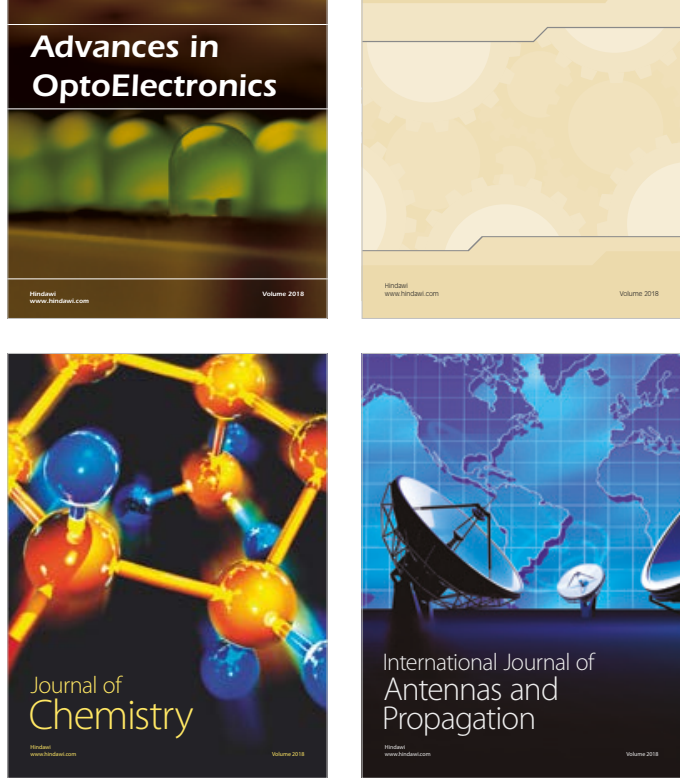

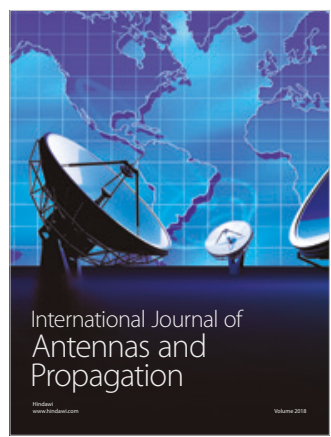

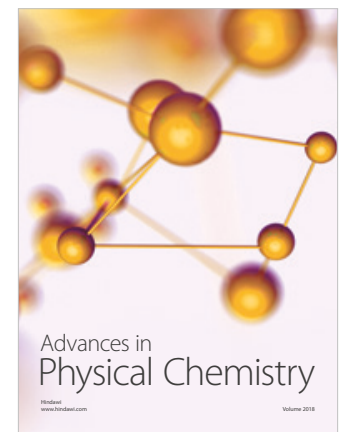

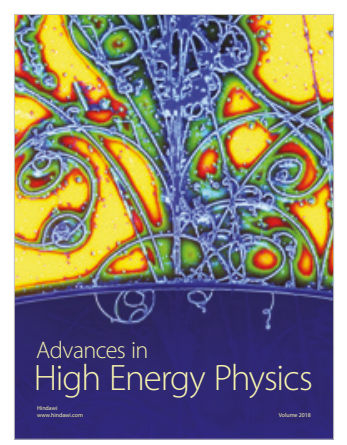

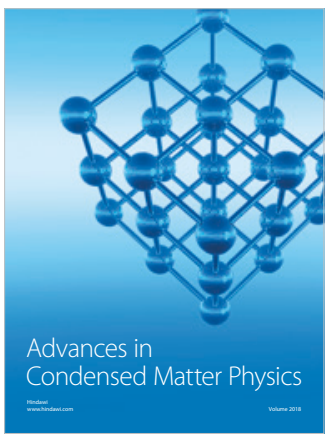

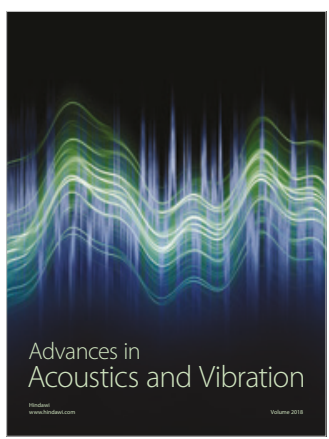

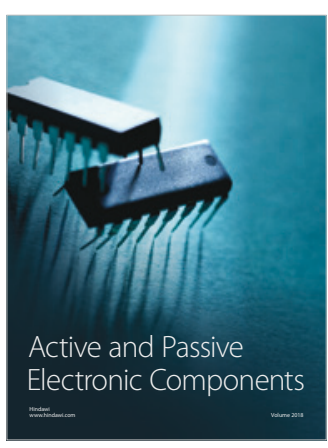
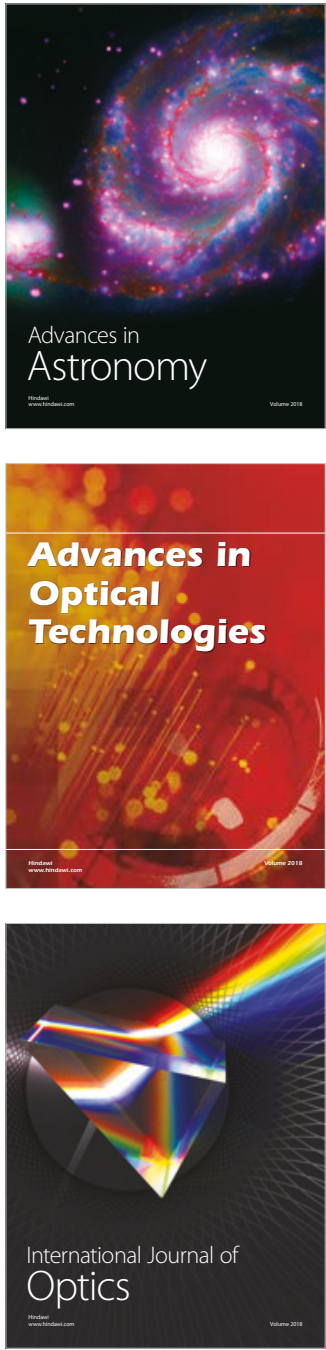